\title{
Brachial plexus injury mimicking a spinal-cord injury
}

Authors Luke J Macyszyn, Ernesto Gonzalez-Giraldo, Michael Aversano, Gregory G Heuer, Eric L Zager, James M Schuster

Institution Department of Neurosurgery, University of Pennsylvania Medical Center, Philadelphia, PA, USA

\section{ABSTRACT}

Objective: High-energy impact to the head, neck, and shoulder can result in cervical spine as well as brachial plexus injuries. Because cervical spine injuries are more common, this tends to be the initial focus for management. We present a case in which the initial magnetic resonance imaging (MRI) was somewhat misleading and a detailed neurological exam lead to the correct diagnosis.

Clinical presentation: A 19-year-old man presented to the hospital following a shoulder injury during football practice. The patient immediately complained of significant pain in his neck, shoulder, and right arm and the inability to move his right arm. He was stabilized in the field for a presumed cervical-spine injury and transported to the emergency department.

Intervention: Initial radiographic assessment (C-spine $\mathrm{CT}$, right shoulder $\mathrm{x}$-ray) showed no bony abnormality. MRI of the cervical-spine showed T2 signal change and cord swelling thought to be consistent with a cord contusion. With adequate pain control, a detailed neurological examination was possible and was consistent with an upper brachial plexus avulsion injury that was confirmed by CT myelogram. The patient failed to make significant neurological recovery and he underwent spinal accessory nerve grafting to the suprascapular nerve to restore shoulder abduction and external rotation, while the phrenic nerve was grafted to the musculocutaneous nerve to restore elbow flexion.

Conclusion: Cervical spinal-cord injuries and brachial plexus injuries can occur by the same high energy mechanisms and can occur simultaneously. As in this case, MRI findings can be misleading and a detailed physical examination is the key to diagnosis. However, this can be difficult in polytrauma patients with upper extremity injuries, head injuries or concomitant spinal-cord injury. Finally, prompt diagnosis and early surgical renerveration have been associated with better long-term recovery with certain types of injury. 


\section{INTRODUCTION}

The high volume of brachial plexus injuries resulting from car and motorcycle accidents has fomented interest in the field of peripheral nerve repair [1]. Such injuries, which mainly occur in young adults [2], can result from direct injury to the nerves or from traction/avulsion of cervical nerve roots from the spinal cord [1]. This is the same population at highest risk for cervical spinal-cord injuries, and the mechanisms of injury can be similar. The diagnosis of a brachial plexus injury is best made by physical examination but this can be difficult in polytrauma patients, and initial imaging studies can be misleading. Tremendous progress in microsurgical techniques, enhanced knowledge of brachial plexus anatomy, increased precision of diagnostic studies, coupled with improved nerve grafting techniques as well as increased availability of donor nerves have eased some of the apprehension with which peripheral nerve repair has been approached in the recent past [3,4].

\section{HISTORY}

A 19-year-old healthy man presented after a high-speed injury to the right shoulder and right upper chest during football practice. The patient immediately complained of excruciating neck, shoulder, and arm pain and inability to move his arm.

The patient was seen at an outside institution where initial radiographic assessment included cervical-spine CT, shoulder films (both without abnormality), and a cervical-spine MRI that showed cord edema and swelling.

Fig 1 Fat suppressed magnetic resonance imaging demonstrating edema in the cervical spinal cord as a result of root avulsion.

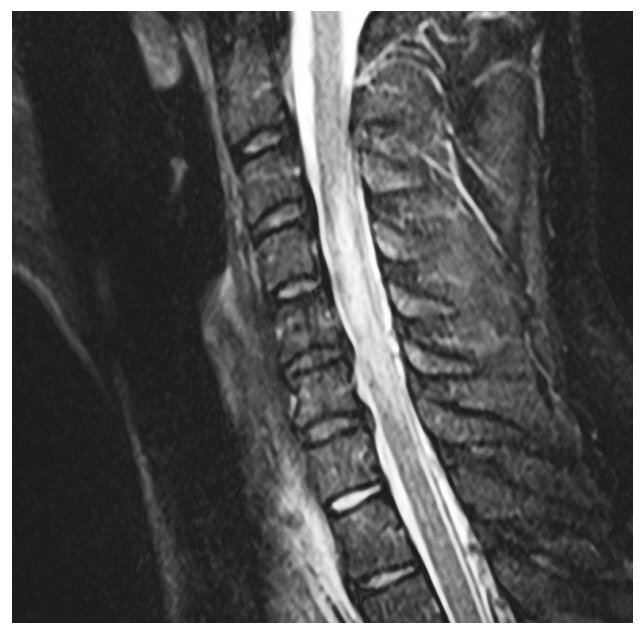

The patient was transferred to our institution for management of possible spinal cord injury.

Physical examination demonstrated right-sided paralysis of the deltoid, biceps, and triceps. The patient was also unable to abduct the shoulder or extend the wrist; he had decreased opposition of the thumb, decreased ulnar motion, decreased abduction and adduction of the digits, as well as decreased grip strength. Additionally, he exhibited C5-7 sensory loss and C8-Tl distribution hypoesthesia.

Further review of the MRI conducted after the incident showed evidence of prevertebral swelling secondary to a tear of the anterior longitudinal ligament and the longus colli, generalized swelling of the spinal cord with moderate increase in $\mathrm{T} 2$ signal from $\mathrm{C} 3-7$, and findings consistent with cervical root avulsions at C5, C6, and C7 (Fig 1). A subsequent CT myelogram was performed, which demonstrated a right pseudomeningocele consistent with cervical root avulsion (Fig 2).

Because of the presence of nerve avulsion and a low likelihood of spontaneous recovery the patient underwent surgery 20 days after the incident. A standard supraclavicular approach was used. The phrenic nerve was found to be intact. The musculocutaneous branch was located and isolated. An $8 \mathrm{~cm}$ long sural-nerve graft was obtained from the right leg and used to connect the phrenic nerve to the cut end of the musculocutaneous nerve. Next, the suprascapular nerve was located. A functional segment of the spinal accessory nerve was found along the posterior margin and this nerve was taken distally and connected to the cut end of the suprascapular nerve. At short-term follow-up there was slight contraction of the triceps and trapezius. Physical therapy and pain management were continued, and electromyography (EMG)/nerve conduction velocity (NCV) muscle testing was scheduled for 6 months after surgery.

Fig 2 Plain film myelography demonstrating a classic pseudomeningocele extending distally from the origin of the right cervical roots to the brachial plexus.

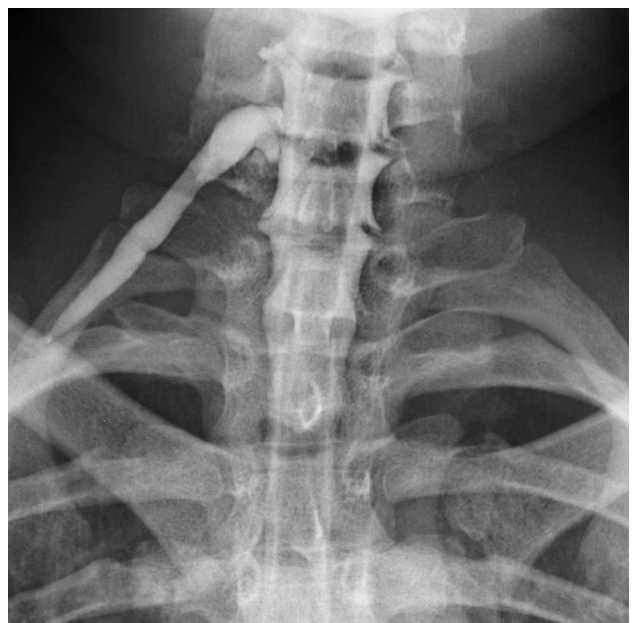




\section{DISCUSSION}

The recovery of shoulder abduction, external rotation, and elbow flexion are the primary goals of surgical reconstruction following injury to the upper brachial plexus (C5-7). The goal of surgery is to restore the patient's ability to fully flex at the elbow and obtain at least $90^{\circ}$ of shoulder abduction and $110^{\circ}$ of external rotation [2]. A patient's capability of performing the activities of daily life is greatly enhanced when these goals are met.

Determining the type, level, and extent of the brachial plexus injury is crucial for the selection of patients who can improve with surgical reconstruction. Preganglionic root injuries represent $70 \%$ of new brachial plexus injuries and are usually addressed with nerve transfers, pedicle muscle transfers, and functioning free-muscle transplantations [4]. The differentiation between root rupture versus avulsion is especially important. Many authors report that the presence of pseudomeningoceles, on either plain film or CT myelography, is almost diagnostic for brachial plexus root avulsion [5]. There are a number of parameters that have been shown to impact the success of surgical reconstruction after brachial plexus injury. The delay between injury and nerve repair is particularly important: delays past 6 months can significantly reduce postoperative improvement $[1,6]$. A number of studies have shown that significant gain in function at the shoulder and elbow may be expected in $80 \%$ of patients if nerve graft or transfer is done within 3 weeks of the onset of injury [3]. Patient adherence to a regimen of occupational and physical therapy can help prevent arthrosis, which can reduce the benefits of muscle renerveration $[1,6]$.

\section{CONCLUSION}

The correct diagnosis of brachial plexus injury can lead to early renerveration and possibly better long term outcome. A high index of suspicion and a detailed neurologic exam is key to the diagnosis especially in the setting of confounding factors such as variable radiographic interpretation, and the presence of other injuries.

\section{REFERENCES}

1. Samii M, Carvalho GA, Nikkhah G, et al (1997) Surgical reconstruction of the musculocutaneous nerve in traumatic brachial plexus injuries. J Neurosurg; 87(6):881-886.

2. Bertelli JA, Ghizoni MF (2006) Concepts of nerve regeneration and repair applied to brachial plexus reconstruction. Microsurgery; 26(4):230-244.

3. Songcharoen $\mathbf{P}$ (2008) Management of brachial plexus injury in adults. Scand J Surg; 97(4):317-323.

4. Chuang DC (2009) Adult brachial plexus reconstruction with the level of injury: review and personal experience. Plast Reconstr Surg; 124(6 Suppl):e359-369.

5. Terzis JK, Kostopoulos VK (2007) The surgical treatment of brachial plexus injuries in adults. Plast Reconstr Surg; 119(4):73e-92e.

6. Schessler MJ, McClellan WT (2010) The role of nerve transfers for $\mathrm{C} 5-\mathrm{C} 6$ brachial plexus injury in adults. WV Med J; 106(1):12-17.

\section{EDITORIAL STAFF PERSPECTIVE}

The preceding case and the discussion both provide an excellent platform to discuss the subject of combination of circumstances, which make the reduction of medicine to an exercise in applying 'checklists' so very unappealing for an affected individual. This patient presented on first and second glances seemingly with a cord contusion. Without the keen observations of the treating physicians, it is very likely that this patient would have been placed into a category of "incomplete cord injury" and not received the optimal treatment for his specific condition, which was a brachial plexus injury with cord contusion. Such a rare combination of circumstances remains the domain of case reports - to share with a larger audience the insights gained from this particular instance. This case also reflects the exact reason why medicine retains an aura of art along with science - to make the leap from the obvious to the actual evidence takes experience and attention to detail paired with knowledge on the part of the practitioner. 